\title{
The molecular basis of T cell acute lymphoblastic leukemia
}

\author{
Pieter Van Vlierberghe ${ }^{1}$ and Adolfo Ferrando ${ }^{1,2,3}$
}

${ }^{1}$ Institute for Cancer Genetics, ${ }^{2}$ Department of Pathology, and ${ }^{3}$ Department of Pediatrics, Columbia University Medical Center, New York, New York, USA.

\begin{abstract}
T cell acute lymphoblastic leukemias (T-ALLs) arise from the malignant transformation of hematopoietic progenitors primed toward $\mathrm{T}$ cell development, as result of a multistep oncogenic process involving constitutive activation of NOTCH signaling and genetic alterations in transcription factors, signaling oncogenes, and tumor suppressors. Notably, these genetic alterations define distinct molecular groups of T-ALL with specific gene expression signatures and clinicobiological features. This review summarizes recent advances in our understanding of the molecular genetics of T-ALL.
\end{abstract}

T cell acute lymphoblastic leukemias (T-ALLs) are aggressive hematologic tumors resulting from the malignant transformation of $\mathrm{T}$ cell progenitors. T-ALL accounts for $10 \%-15 \%$ of pediatric and $25 \%$ of adult ALL cases (1) and is characteristically more frequent in males than females. Clinically, T-ALL patients show diffuse infiltration of the bone marrow by immature $T$ cell lymphoblasts, high white blood cell counts, mediastinal masses with pleural effusions, and frequent infiltration of the central nervous system at diagnosis. Although originally associated with high relapse rates, the prognosis of T-ALL has gradually improved with the Introduction of intensified chemotherapy, with cure rates in modern protocols reaching over $75 \%$ in children and about $50 \%$ in adults with this disease (2). However, the outcome of T-ALL patients with primary resistant or relapsed leukemia remains poor $(3,4)$. Therefore, current research efforts are focused on the search for targets for the development of more effective and less toxic antileukemic drugs (5), which will likely require a greater degree of specificity and an improved understanding of the molecular events that lead to the disease.

$\mathrm{T}$ cell transformation is a multi-step process in which different genetic alterations cooperate to alter the normal mechanisms that control cell growth, proliferation, survival, and differentiation during thymocyte development. In this context, constitutive activation of NOTCH1 signaling is the most prominent oncogenic pathway in $\mathrm{T}$ cell transformation (6). However, deletions of the CDKN2A locus in chromosome band 9p21, which encompasses the $p 16 / I N K 4 A$ and $p 14 / A R F$ suppressor genes, are present in more than $70 \%$ of all T-ALL cases $(1,7)$. Thus, constitutive activation of NOTCH signaling cooperates with loss of $p 16 / I N K 4 A$ and $p 14 / A R F$ in $\mathrm{T}$ cell transformation and constitutes the core of the oncogenic program in the pathogenesis of T-ALL.

In addition, T-ALLs characteristically show the translocation and aberrant expression of transcription factor oncogenes. These chromosomal rearrangements place T-ALL transcription factor oncogenes under the control of strong $\mathrm{T}$ cell-specific enhancers located in the TCRB (7q34) or TCRA-TCRD (14q11) loci, resulting in their aberrant expression in $\mathrm{T}$ cell progenitors. These oncogenic transcription factors include basic helix-loop-helix (bHLH) family members such as TAL1 (8-10), TAL2 (11), LYL1 (12), and BHLHB1 (13); LIM-only domain (LMO) genes such as LMO1 and LMO2 (14-16); the TLX1/HOX11 (17-19), TLX3/HOX11L2 (1, 20),

Conflict of interest: The authors have declared that no conflict of interest exists. Citation for this article: J Clin Invest. 2012;122(10):3398-3406. doi:10.1172/JCI61269.
NKX2.1 (21), NKX2.2 (21), NKX2.5 (22), and HOXA homeobox (HOX) genes (23, 24); MYC (25); MYB (26); and TAN1, a truncated and constitutively activated form of the NOTCH1 receptor (27). In addition, some of these $\mathrm{T}$ cell transcription factor oncogenes can be activated as result of alternative genetic rearrangements. Most notably, the TLX3/HOX11L2 locus is recurrently translocated to T cell regulatory sequences in the proximity of the BCL11B locus and only rarely translocated to the TCR loci (20). In addition, small intrachromosomal deletions in chromosome $1 \mathrm{p} 32$ result in TAL1 overexpression (28), and cryptic deletions in chromosome 11p13 can lead to activation of the LMO2 oncogene (29).

Importantly, gene expression profiling studies have revealed a limited number of well-defined molecular groups of T-ALL $(1,21$, $23,30)$, which share unique gene expression signatures reflecting distinct stages of arrest during T cell development (1). Early immature T-ALLs show an early block at the double-negative stage of thymocyte development $(1,31,32)$. In contrast, early cortical T-ALLs are characteristically CD1a, CD4, and CD8 positive and are typically associated with activation of the TLX1, TLX3, NKX2.1, and NKX2.2 homeobox genes $(1,21)$. Finally, late-cortical thymocyte T-ALLs express CD4, CD8, and CD3 and show activation of the TAL1 transcription factor oncogene (1). Table 1 provides an overview of all main driving genetic lesions that characterize these unique molecular genetic T-ALL subtypes.

The diversity of genetic lesions involved in the pathogenesis of T-ALL is further complicated by a number of recurrent cytogenetic and molecular alterations that are common between all molecular subtypes and cause deregulation in specific cellular processes, including cell cycle signaling, cell growth and proliferation, chromatin remodeling, $\mathrm{T}$ cell differentiation, and self-renewal (Table 2).

Recent studies have linked the early immature T-ALL group with the activation of a transcriptional program related to hematopoietic stem cells and myeloid progenitors $(32,33)$, aberrant expression of the MEF2C gene (21), mutations in acute myeloid leukemia oncogenes and tumor suppressors $(32,33)$ and inactivation of important transcription factors such as RUNX1, GATA3, and ETV6 $(32,33)$. Notably, these tumors frequently show absence of biallelic TCR $\gamma$ deletions (34) and are associated with a very poor prognosis $(31,34)$.

\section{Constitutive activation of NOTCH1 signaling in T-ALL}

The NOTCH signaling pathway plays a critical role in cell lineage commitment decisions during development (ref. 35 and Figure 1). Aberrant NOTCH1 signaling was originally linked to the patho- 


\section{Table 1}

Genetic lesions that define molecular-genetic subtypes in T-ALL

\begin{tabular}{|c|c|c|c|c|}
\hline Gene category & Gene target & Genetic rearrangement & Outcome (ref.) & Frequency ${ }^{A}$ (ref.) \\
\hline \multirow[t]{6}{*}{ bHLH family members } & TAL1 & $\mathrm{t}(1 ; 14)(\mathrm{p} 32 ; \mathrm{q} 11)$ & Good (66-68) & $3 \%(28)$ \\
\hline & & $\mathrm{t}(1 ; 7)(\mathrm{p} 32 ; \mathrm{q} 34)$ & Good (66-68) & $3 \%(28)$ \\
\hline & & 1 p32 deletion & Good (66-68) & $16 \%-30 \%(61)$ \\
\hline & TAL2 & $\mathrm{t}(7 ; 9)(\mathrm{q} 34 ; \mathrm{q} 32)$ & NA & $1 \%(11,30)$ \\
\hline & $L Y L 1$ & $\mathrm{t}(7 ; 19)(q 34 ; p 13)$ & NA & $1 \%(12)$ \\
\hline & $B H L H B 1$ & $\mathrm{t}(14 ; 21)(\mathrm{q} 11.2 ; \mathrm{q} 22)$ & NA & $1 \%(13)$ \\
\hline \multirow[t]{6}{*}{ LMO family members } & LMO1 & $\mathrm{t}(11 ; 14)(\mathrm{p} 15 ; \mathrm{q} 11)$ & NA & $1 \%(15,30)$ \\
\hline & & $\mathrm{t}(7 ; 11)(\mathrm{q} 34 ; \mathrm{p} 15)$ & NA & $1 \%(15,30)$ \\
\hline & LMO2 & $\mathrm{t}(11 ; 14)(\mathrm{p} 13 ; q 11)$ & NA & $6 \%(126)$ \\
\hline & & $\mathrm{t}(7 ; 11)(q 34 ; p 13)$ & NA & $6 \%(126)$ \\
\hline & & $11 p 13$ deletion & NA & $3 \%(29)$ \\
\hline & LMO3 & $\mathrm{t}(7 ; 12)(\mathrm{q} 34 ; \mathrm{p} 12)$ & NA & $<1 \%(21)$ \\
\hline \multirow[t]{13}{*}{ Homeobox family members } & $T L X 1$ & $\mathrm{t}(11 ; 14)(\mathrm{p} 15 ; q 11)$ & Good (76) & $\begin{array}{c}5 \%-10 \%(126) \\
30 \%(76,88)\end{array}$ \\
\hline & $T L X 3$ & $\mathrm{t}(11 ; 14)(\mathrm{p} 15 ; q 11)$ & Poor $(83,88,126)$ & $20 \%(20)$ \\
\hline & & & No impact (67) & $5 \%(84)$ \\
\hline & & & Good (127) & $5 \%(84)$ \\
\hline & HOXA & $\operatorname{Inv}(7)(\mathrm{p} 15 \mathrm{q} 34)$ & NA & $3 \%(23,128)$ \\
\hline & & $\mathrm{t}(7 ; 7)(\mathrm{p} 15 ; \mathrm{q} 34)$ & NA & $3 \%(23,128)$ \\
\hline & HOXA (CALM-AF10) & $\mathrm{t}(10 ; 11)(\mathrm{p} 13 ; \mathrm{q} 14)$ & Poor $(87,126)$ & $5 \%-10 \%(87)$ \\
\hline & HOXA (MLL-ENL) & $\mathrm{t}(11 ; 19)(q 23 ; p 13)$ & NA & $1 \%(88)$ \\
\hline & HOXA (SET-NUP214) & $9 q 34$ deletion & No impact (33) & $3 \%(30)$ \\
\hline & & $\operatorname{inv}(14)(q 11.2 q 13)$ & NA & \\
\hline & NKX2.1 & $\operatorname{inv}(14)(q 13 q 32.33)$ & NA & $5 \%(21)$ \\
\hline & & $\mathrm{t}(7 ; 14)(\mathrm{q} 34 ; \mathrm{q} 13)$ & NA & \\
\hline & $N K X 2.2$ & $\mathrm{t}(14 ; 20)(\mathrm{q} 11 ; \mathrm{p} 11)$ & NA & $1 \%(21)$ \\
\hline Proto-oncogene & $C-M Y B$ & $\mathrm{t}(6 ; 7)(\mathrm{q} 23 ; \mathrm{q} 34)$ & NA & $3 \%(26)$ \\
\hline
\end{tabular}

${ }^{A}$ Frequency refers to the frequency in which each mutation occurs in the patient population.

genesis of T-ALL by the cloning of the $t(7 ; 9)(q 34 ; q 34.3)$ chromosomal translocation, which leads to the expression of a truncated and constitutively active form of NOTCH1 (27). However, the central role of NOTCH1 in T cell transformation was only realized upon the identification of activating mutations in the NOTCH1 gene present in over $50 \%$ of T-ALL cases (6). NOTCH1 mutations typically involve specific domains responsible for controlling the initiation and termination of $\mathrm{NOTCH}$ signaling $(6,36)$. In addition FBXW7 mutations, present in about $15 \%$ of T-ALL cases, contribute to NOTCH activation by impairing the proteasomal degradation of activated NOTCH1 in the nucleus (refs. 37, 38, and Figure 2).

The identification of activating NOTCH1 mutations, present in nearly $60 \%$ of T-ALL patients, has created enormous interest in developing molecularly tailored therapies for T-ALL (6). Most notably, $\gamma$-secretase inhibitors (GSIs), which block the proteolytic cleavage of the NOTCH receptors and preclude the release of activated NOTCH1 (ICN1) from the membrane, have been proposed as potential targeted therapy in T-ALL $(6,39-42)$. More recently, stapled peptides targeting the NOTCH transcriptional complex and NOTCH1-specific inhibitory antibodies have been proposed as alternative anti-NOTCH1 therapies for the treatment of T-ALL (refs. 43, 44, and Figure 1).

A number of studies have recently addressed the prognostic significance of NOTCH1 and FBXW7 mutations in T-ALL (45-51). Overall, these studies show that NOTCH activation is associated with improved early therapeutic response and increased sensitivity to glucocorticoids. However, this early benefit only translates into improved overall survival in some studies (45-48). The asso- ciation of NOTCH1 mutations with increased glucocorticoid response is particularly intriguing given that expression of activated NOTCH1 can impair glucocorticoid-induced cell death in thymocytes (52) and that blocking NOTCH1 signaling with GSIs can reverse glucocorticoid resistance in some T-ALLs (53). This last notion was recently tested in a preclinical setting using a combination of PF-03084014, a clinically-relevant GSI, and dexamethasone in glucocorticoid-resistant T-ALL. The study revealed that this glucocorticoid/GSI combination has a synergistic antileukemic effect in human T-ALL cell lines, primary human T-ALL patient samples, and in an in vivo mouse xenograft model of T-ALL (54).

\section{Alterations in cell cycle regulators}

The CDKN2A locus in the short arm of chromosome 9 contains the $p 16 I N K 4 A$ and $p 14 A R F$ tumor suppressor genes. P16INK4A directly blocks cyclin D-CDK4/6 complexes, whereas p14ARF inhibits MDM2, a negative regulator of the TP53 oncoprotein $(55,56)$. CDKN2A deletions are the most frequent abnormality in T-ALL, present in over $70 \%$ of patients (7). In addition, the $t(12 ; 14)$ (p13;q11) and $\mathrm{t}(7 ; 12)(\mathrm{q} 34 ; \mathrm{p} 13)$ translocations can induce aberrantly high levels of expression of the CCND2 cell cycle regulator (57) and chromosomal deletions recurrently inactivate $R B 1$ and CDKN1B in some T-ALL cases $(58,59)$.

The prognostic implications of loss of heterozygosity at the short arm of chromosome 9 were evaluated in pediatric T-ALL patients treated according to the Berlin-Frankfurt-Munster regimen (60). This study showed that loss of heterozygosity at $9 \mathrm{p}$ was associated with a favorable initial treatment response in T-ALL (60). 
Table 2

Classification of other recurrent genetic alterations in T-ALL

\begin{tabular}{|c|c|c|c|c|}
\hline Category & Gene target & Genetic rearrangement & Outcome (ref.) & Frequency (ref.) \\
\hline \multirow[t]{2}{*}{ NOTCH1 pathway } & NOTCH1 & $\begin{array}{l}\mathrm{t}(7 ; 9)(q 34 ; \mathrm{p} 13) \\
\text { Activating mutation }\end{array}$ & $\begin{array}{c}\text { NA } \\
\text { Good }(51,129) \\
\text { GPR }(45,49,50) \\
\text { No impact }(48)\end{array}$ & $\begin{array}{l}<1 \%(27) \\
>60 \%(6)\end{array}$ \\
\hline & FBXW7 & Inactivating mutation & NA & $8 \%-30 \%(37,38)$ \\
\hline \multirow[t]{3}{*}{ Cell cycle defects } & $\begin{array}{l}\text { CDKN2A/2B } \\
\text { CCND2 }\end{array}$ & $\begin{array}{c}\text { 9p21 deletion methylation } \\
\mathrm{t}(7 ; 12)(\mathrm{q} 34 ; \mathrm{p} 13) \\
\mathrm{t}(12 ; 14)(\mathrm{p} 13 ; \mathrm{q} 11)\end{array}$ & $\begin{array}{l}\text { Good }(60) \\
\text { NA }\end{array}$ & $\begin{array}{l}70 \%(7) \\
1 \%(57)\end{array}$ \\
\hline & $R B 1$ & $13 q 14$ deletion & No impact (33) & $4 \%(58)$ \\
\hline & CDKN1B & 12 p13 deletion & NA & $2 \%(59)$ \\
\hline \multirow{7}{*}{$\begin{array}{l}\text { Cell growth transcription factor } \\
\text { tumor suppressors }\end{array}$} & $M Y C$ & $\mathrm{t}(8 ; 14)(\mathrm{q} 24 ; \mathrm{q} 11)$ & NA & $1 \%(25)$ \\
\hline & WT1 & Inactivating mutation/deletion & No impact (80) & $10 \%(80)$ \\
\hline & LEF1 & Inactivating mutation/deletion & NA & $10 \%-15 \%(93)$ \\
\hline & ETV6 & Inactivating mutation/deletion & No impact (33) & $13 \%(32,33)$ \\
\hline & $B C L 11 B$ & Inactivating mutation/deletion & No impact (33) & $10 \%(98)$ \\
\hline & RUNX1 & Inactivating mutation/deletion & $\begin{array}{l}\text { No impact (33) } \\
\text { Poor (101) }\end{array}$ & $10 \%-20 \%(100,101)$ \\
\hline & GATA3 & Inactivating mutation/deletion & Poor (33) & $5 \%(33)$ \\
\hline \multirow[t]{13}{*}{ Signal transduction } & PTEN & Inactivating mutation & No impact $(106,130)$ & $10 \%(33,106,130)$ \\
\hline & & $10 q 23$ deletion & Poor (33) & $10 \%(33,106,130)$ \\
\hline & NUP214-ABL1 & Episomal 9q34 amplification & $\begin{array}{c}\text { Poor (108) } \\
\text { No impact (131) }\end{array}$ & $4 \%(108)$ \\
\hline & $E M L 1-A B L 1$ & $t(9 ; 14)(q 34 ; q 32)$ & NA & $<1 \%(109)$ \\
\hline & ETV6-ABL1 & $\mathrm{t}(9 ; 12)(q 34 ; p 13)$ & NA & $<1 \%(110)$ \\
\hline & $B C R-A B L 1$ & $\mathrm{t}(9 ; 22)(q 34 ; q 11)$ & Poor (132) & $<1 \%(132)$ \\
\hline & NRAS & Activating mutation & No impact (33) & $5 \%-10 \%(32,113)$ \\
\hline & NF1 & Inactivating mutation/deletion & No impact (33) & $3 \%(114)$ \\
\hline & JAK1 & Activating mutation & No impact (33) & $4 \%-18 \%(118,119)$ \\
\hline & ETV6-JAK2 & $\mathrm{t}(9 ; 12)(\mathrm{p} 24 ; \mathrm{p} 13)$ & NA & $<1 \%(117)$ \\
\hline & JAK3 & activating mutation & No impact (33) & $5 \%(33)$ \\
\hline & FLT3 & activating mutation & No impact (33) & $2 \%-4 \%(133,134)$ \\
\hline & IL7R & activating mutation & No impact (33) & $10 \%(120,121)$ \\
\hline \multirow[t]{4}{*}{ Chromatin remodeling } & $E Z H 2$ & Inactivating mutation/deletion & Poor (33) & $10 \%-15 \%(33,135)$ \\
\hline & SUZ12 & Inactivating mutation/deletion & No impact (33) & $10 \%(33)$ \\
\hline & EED & Inactivating mutation/deletion & No impact (33) & $10 \%(33)$ \\
\hline & PHF6 & Inactivating mutation/deletion & No impact (81) & $20 \%-40 \%(81)$ \\
\hline
\end{tabular}

GPR, good prednisone response.

\section{T-ALL-specific transcription factor oncogenes}

Class II bHLH transcription factors. Overexpression and aberrant activation of a number of bHLH transcription factors activated by chromosomal translocations is a frequent oncogenic event in T-ALL. These proteins are characterized by a basic domain that mediates DNA binding and two $\alpha$-helices connected by a loop that are involved in the formation of homodimeric and heterodimeric complexes. The TAL1 gene in chromosome band $1 \mathrm{p} 32$ is a key regulator of hematopoietic stem cell development expressed in hematopoietic progenitors, mast cells, and erythroid and megakaryocyte progenitors (5). Abnormal expression of TAL1 is present in approximately $60 \%$ of T-ALL cases as a result of various chromosomal rearrangements $(1,61)$. Thus, in $3 \%$ of childhood T-ALL, the translocation $\mathrm{t}(1 ; 14)(\mathrm{p} 32 ; \mathrm{q} 11)$ places $T A L 1$ gene expression under the control of TCRA/D enhancers. In addition, $16 \%-30 \%$ of T-ALLs harbor a small intrachromosomal rearrangement that places TAL1 under the control of the promoter of the neighbor gene STIL, which is highly expressed in T cells (28). T-ALL cases with TAL1 expression show developmental arrest at the late double-positive stage of thymocyte development (1). In addition, LYL1, TAL2, and $B H L H B 1$, three genes encoding bHLH factors closely related to $T A L 1$, are translocated and aberrantly expressed in rare cases of T-ALL (11-13). The oncogenic potential of TAL1 is illustrated by the induction of T-ALL in transgenic mice expressing TAL1 in developing thymocytes $(62,63)$. In T-ALL cells, TAL1 forms primarily inactive transcriptional complexes that contain $\mathrm{E}$ proteins and the LMO factors, LMO1 and LMO2, resulting in decreased expression of E protein target genes. Thus, although TAL1 may be present in transcriptional complexes activating the expression of some target genes (64), it has been proposed that the oncogenic activity of TAL1 could be mediated primarily by reducing the level of transcriptional activity of promoters normally controlled by $\mathrm{E}$ proteins (65). From a clinical point of view, a number of studies have suggested that late-cortical thymocyte T-ALLs showing TAL1 gene rearrangements have a more favorable outcome (66-68).

LMO proteins. LIM domain proteins were originally linked with T-ALL in cases harboring the $\mathrm{t}(11 ; 14)(\mathrm{p} 15 ; \mathrm{q} 11)$ and $\mathrm{t}(11 ; 14)$ (p13;q11) chromosomal translocations involving the LMO1 and 


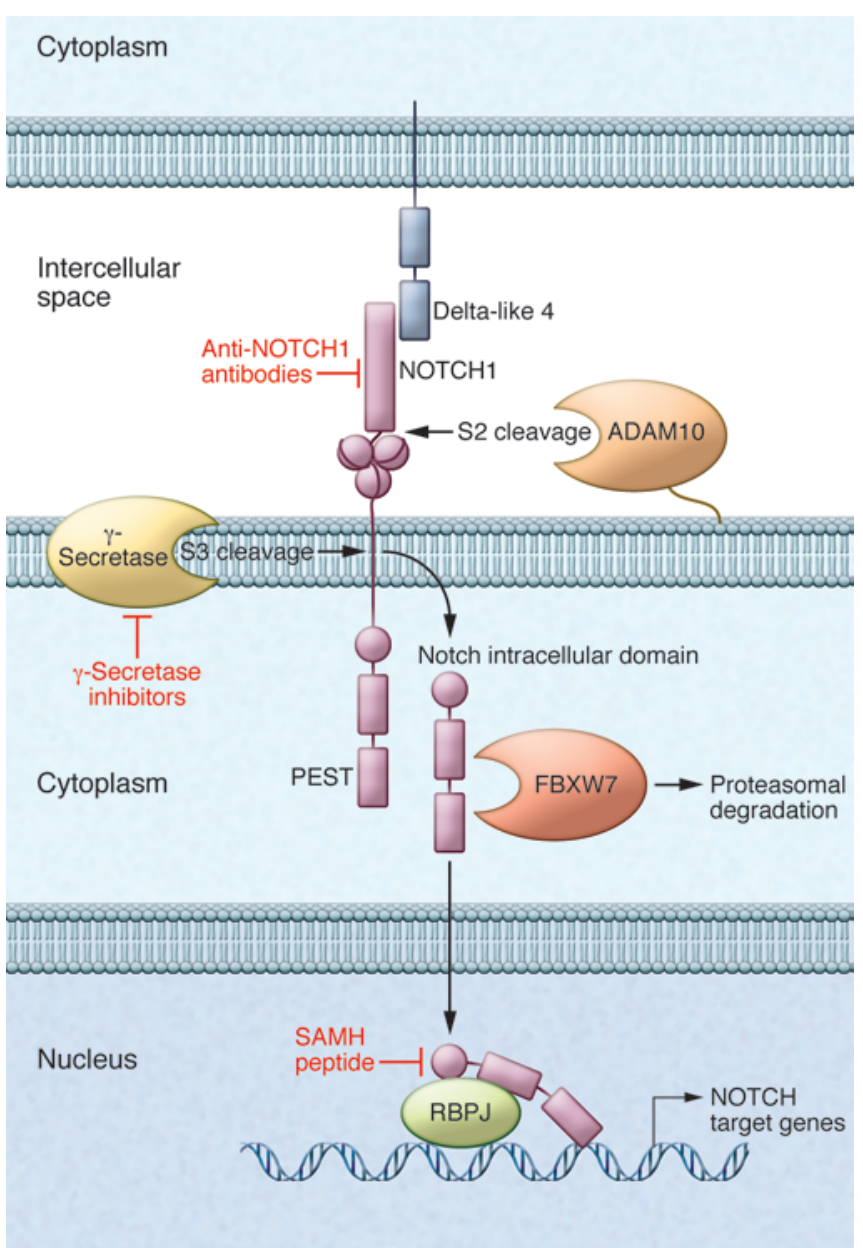

LMO2 genes, respectively (14-16). Although these translocations only occur in $9 \%$ of pediatric T-ALL, aberrant LMO2 expression can be found in up to $45 \%$ of T-ALL cases, suggesting additional mechanisms of activation. Unlike bHLH proteins, LMO1 and LMO2 do not interact directly with DNA; but they form transcriptional complexes with TAL1 and LYL1 $(1,69)$. Consistently, activation of LMO1 and LMO2 is most frequently found in cases with deregulated TAL1 and/or LYL1 expression (1). In line with this notion, the oncogenic activity of $L m o 1$ or $L m o 2$ in transgenic mice $(70,71)$ was shown to be enhanced in double-transgenic animals expressing TAL1 in developing thymocytes $(69,72)$.

Although many of the genetic programs controlled by oncogenic LMO factors remain to be elucidated, a recent report from McCormack and coworkers has shown that forced expression of Lmo2 in mouse $\mathrm{T}$ cell progenitor cells confers self-renewal capacity to this normally non-self-renewing population (73). These results suggest a close relationship between the activation of stem cell properties and the oncogenic effects of LMO2 in T cell precursors (73). However, this model does not seem to reflect the late-cortical immunophenotypic arrest observed in primary LMO2-rearranged human T-ALL samples. LMO2 rearrangements predicted poor outcome in a stratified analysis of pediatric T-ALL patients (29). However, given the small number of patients that harbor this specific genetic rearrangement, the significance of these findings needs to be validated in larger and independent T-ALL patient cohorts.

\section{Figure 1}

Schematic representation of $\mathrm{NOTCH} 1$ signaling in $\mathrm{T}$ cell progenitors. Interaction of the NOTCH ligand delta-like 4, expressed on the surface of thymic stroma cells, with NOTCH1 triggers a double proteolytic cleavage of the receptor in T cell progenitors first by the ADAM10 metalloprotease and subsequently by the gamma secretase complex. Release of the intracellular domains of NOTCH1 from the membrane activates the expression of NOTCH target genes in the nucleus. FBXW7 recognizes the PEST domain of activated NOTCH1 and terminates NOTCH signaling in the proteasome. Inhibition of ADAM10 cleavage with anti-NOTCH 1 inhibitory antibodies, blockage of $\gamma$-secretase activity with small-molecule inhibitors, and disruption of the NOTCH1 nuclear transcriptional complex with small SAMH1 peptides are all approaches to effectively block NOTCH1 signaling in T-ALL.

HOX transcription factor oncogenes TLX1, TLX3, and HOXA. HOX transcription factors play essential roles in body patterning and organogenesis during development. TLX1 is the founding member of a family of HOX genes that includes TLX2 and TLX3 (74). TLX1 was originally identified as the gene translocated into the TCRA/D locus in the recurrent $\mathrm{t}(10 ; 14)(\mathrm{q} 24 ; \mathrm{q} 11)$ in T-ALL $(17-19,75)$. This genetic rearrangement induces aberrant TLX1 expression and occurs in about $5 \%-10 \%$ of pediatric and $30 \%$ of adult T-ALLs $(1,76)$. Recently, the leukemogenic role of TLX1 in T cell transformation has been firmly established using a TLX1 transgenic mouse model that developed clonal T cell leukemias (77). Notably, TLX1-induced T-ALLs in mice shared a common transcriptional program with TLX1-positive human tumors characterized by the downregulation of TLX1 direct target genes.

$T L X 3$ is overexpressed in $20 \%-25 \%$ of pediatric and $5 \%$ of adult T-ALLs harboring the $t(5 ; 14)(\mathrm{q} 35 ; \mathrm{q} 32)$ translocation $(20)$. This rearrangement places the TLX3 oncogene under the control of strong T cell regulatory elements in the BCL11B locus (20). Currently, the in vivo role of aberrant $T L X 3$ expression in the pathogenesis of T-ALL remains to be established.

TLX1 and TLX3 leukemias share an overlapping mechanism of transformation, have convergent gene expression signatures, and show specific cooperating mutations rarely present in nonTLX-induced leukemias, including the NUP214-ABL1 fusion oncogene and mutations in the PTPN2, Wilms tumor 1 (WT1), and $P H F 6$ tumor suppressors (78-81). In addition, it was recently proposed that the unique cortical thymic maturation arrest in TLX-induced tumors may be related to the binding of TLX1/ TLX3-ETS1 complexes to TCRA enhancer sequences, with the consequent downregulation of TCRA gene rearrangement and expression (82). TLX1 expression has been linked with a favorable prognosis and a low risk of relapse in children and adults $(1,76)$. However, aberrant TLX3 expression is associated with a less favorable prognosis and a higher incidence of relapse in some studies $(83,84)$.

Finally, about $3 \%$ of T-ALL patients harbor translocations in the HOXA cluster of HOX genes in 7p15 into the TCRB and TCRG loci, resulting in aberrant expression of the HOXA10 and HOXA9 genes (23). In addition, chromosomal translocations that generate fusion transcripts encoding chimeric transcription factor oncogenes can also be found in T-ALL. About $5 \%$ of T-ALLs express the MLL-AFF1 (MLL-AF4) and MLL-MLLT1 (MLL-ENL) fusion genes (85). Notably, $M L L$ fusion oncogenes are associated with poor prognosis in precursor B cell ALL, although the $M L L$ MLLT1 fusion rearrangement seems to confer a favorable progno- 


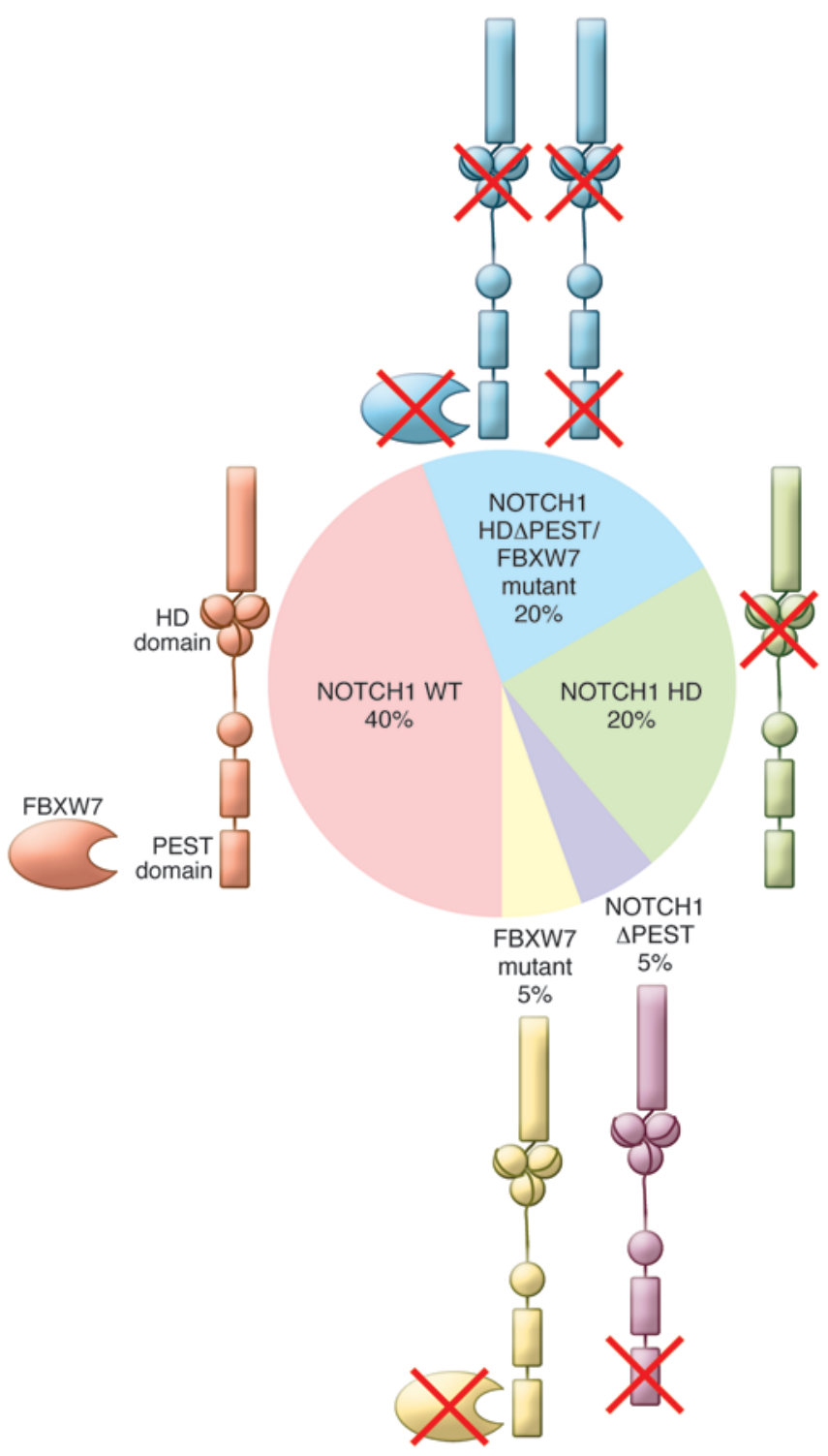

sis in T-ALL $(1,86)$. In addition, the PICALM-MLLT10 fusion oncogene is present in $5 \%-10 \%$ of T-ALLs $(23,87)$, and a rare cryptic $\operatorname{del}(9)(\mathrm{q} 34.11 \mathrm{q} 34.13)$ deletion generating the SET-NUP214 fusion gene in T-ALL has been described (30). A common feature of leukemias harboring these fusion transcription factor oncogenes is the aberrant expression of $H O X A$ genes $(1,23,30,87,88)$, suggesting a more general pathogenic role of HOXA dysregulation in the pathogenesis of T-ALL.

Proto-oncogenes. The MYC oncogene is activated in 1\% of T-ALLs as result of the $\mathrm{t}(8 ; 14)(\mathrm{q} 24 ; \mathrm{q} 11)$ translocation (25). However, MYC is broadly activated in T-ALL and functions as a critical NOTCH1 direct target gene, promoting cell growth and proliferation $(89,90)$. $c-M Y B$, a leucine zipper transcription factor oncogene, is translocated and overexpressed in T-ALL cases with the $\mathrm{t}(6 ; 7)(\mathrm{q} 23 ; \mathrm{q} 32)$. MYB translocations are characteristically found in childhood T-ALLs diagnosed before 2 years of age and show a marked increase in the expression of proliferation and mitosis genes (26). In addition, focal duplications of the MYB locus are present in about $10 \%$ of T-ALLs $(91,92)$.

\section{Figure 2}

Prevalence and mechanisms of aberrant NOTCH1 signaling in T-ALL $(40,125)$. Schematic representation of the $\mathrm{NOTCH} 1$ receptor structure is shown for each type of NOTCH1 mutation found in T-ALL. The resting, membrane-bound domain structure of the $\mathrm{NOTCH} 1$ receptor is shown as depicted and described in Figure 1. Crossed-out domains in NOTCH1 and FBXW7 indicate the targeted areas disrupted in these proteins for each of the NOTCH1 and FBXW7 mutant alleles found in T-ALL.

\section{Loss of transcription factor tumor suppressor genes}

Over the last few years, a wide variety of tumor suppressor genes have been shown to be mutated and deleted in T-ALL. However, the specific role of most of these transcriptional regulators in $\mathrm{T}$ cell transformation remains to be elucidated and is an area of active current investigation.

Deletions and mutations in the WT1 gene are present in about $10 \%$ of T-ALLs $(78,80)$. WT1 mutations found in T-ALL are predominantly heterozygous frameshift mutations resulting in truncation of the C-terminal zinc finger domains of this transcription factor and are frequently associated with oncogenic expression of the TLX1, TLX3, or HOXA oncogenes $(78,80)$.

Monoallelic or biallelic deletions involving the LEF1 locus and mutations in the LEF1 gene are present in about $15 \%$ of T-ALL cases (93). Notably, these leukemias show a characteristic differentiation arrest at the early cortical thymocyte stage of differentiation that resembles that of TLX1-positive tumors (93).

ETV6 encodes an ETS family transcriptional repressor strictly required for the development of hematopoietic stem cells $(94,95)$ and plays a prominent role in precursor B cell ALLs harboring the $t(8 ; 21)$ translocation, which results in the expression of the ETV6-RUNX1 oncogene (96). ETV6 mutations encoding truncated proteins with dominant-negative activity are frequently found in early immature T-ALLs $(32,33)$.

$B C L 11 B$ encodes a transcription factor critically required for normal $\mathrm{T}$ cell development (97). Loss-of-function mutations and heterozygous deletions of the $B C L 11 B$ are recurrently found in T-ALL, suggesting that $B C L 11 B$ haploinsufficiency may be an important pathogenetic event in $T$ cell leukemogenesis $(77,98)$.

RUNX1 is an important transcription factor tumor suppressor gene involved in the pathogenesis of acute myeloid and precursor $\mathrm{B}$ cell leukemias (99). A recent systems biology study that aimed at deciphering the transcriptional regulatory network controlled by $T L X 1$ and TLX3 in the pathogenesis T-ALL revealed a prominent role for RUNX1 in the control of the oncogenic program downstream of the TLX1 and TLX 3 HOX transcription factor oncogenes (100). Moreover, loss-of-function mutations in RUNX1 can be found in immature T-ALL samples, suggesting a tumor suppressor role for RUNX1 in T cell transformation $(33,100,101)$.

GATA3 is an important regulator of T cell differentiation and has a crucial role in the development of early $\mathrm{T}$ cell progenitors (102). Recurrent somatic GATA3 mutations have been exclusively identified in the early immature ETP-T-ALL subtype (33). These GATA3 mutations cluster in the zinc finger DNA-binding protein domain, recurrently involve missense mutations targeting a specific R276 residue critically required for DNA binding (33), and may be responsible for the early block in $\mathrm{T}$ cell development of these leukemias.

\section{Genetic alterations in the chromatin remodeling}

The polycomb repressive complex 2 (PRC2) is the "writer" of a major repressive chromatin modification, histone $\mathrm{H} 3$ lysine 27 
trimethylation (H3K27me3). Two recent studies reported lossof-function mutations and deletions of EZH2 and SUZ12 genes, which encode two critical components of the PRC2 complex, in up to $25 \%$ of T-ALLs $(33,103)$. In addition, NOTCH1 activation was shown to specifically induce loss of the repressive H3K27me3 mark by antagonizing PRC2 complex activity during T cell transformation, suggesting a dynamic interplay between oncogenic NOTCH1 activation and loss of PRC2 function in the pathogenesis of T-ALL (103). Importantly, conditional ablation of Ezh2 in early hematopoietic progenitors using a conditional knockout mouse model revealed a high frequency of spontaneous $\gamma \delta \mathrm{T}$ cell leukemias, further establishing the PRC2 complex as a bona fide tumor suppressor in the pathogenesis of T-ALL (104).

PHF6, a plant homeodomain-containing (PHD-containing) factor with a proposed role in epigenetic regulation of gene expression, is mutated and deleted about $16 \%$ of pediatric and $38 \%$ of adult T-ALL cases (81). Notably, PHF6 is located in Xq26, and PHF6 mutations are almost exclusively found in male patients with T-ALL (81). Proteins harboring similar PHD domains have been implicated in reading methylation marks present at specific histone tail residues, suggesting that loss of PHF6 might influence genome-wide chromatin structure.

\section{Genetic alterations in signal transduction pathways}

In addition to the above mentioned genetic lesions in transcription factors and chromatin regulators, genes encoding critical components of signaling pathways controlling the growth, proliferation, lineage commitment, and survival of $\mathrm{T}$ cell progenitors are frequently mutated in T-ALL.

The PTEN tumor suppressor encodes a critical negative regulator of the PI3K-AKT signaling pathway. PTEN specifically dephosphorylates and inactivates PIP3, the lipid second messenger product of the PI3K complex responsible for recruitment and activation of the AKT1 kinase (105). Deletion mutations in PTEN occur in 5\%-10\% of T-ALL cases, and overall 17\% of T-ALLs lack PTEN protein expression (106).

ABL1 rearrangements occur in about $8 \%$ of T-ALLs (107), and $6 \%$ of T-ALL cases show a complex rearrangement resulting in the episomal amplification and expression of the NUP214-ABL1 fusion oncogene (108). Interestingly, the NUP214-ABL1 rearrangement is almost exclusively found TLX1 and TLX3 T-ALLs (108), which suggests a specific functional interaction between oncogenic ABL1 signaling and TLX1 expression in the pathogenesis of T-ALL. Related ABL1 rearrangements present in T-ALL include EML1-ABL1 and ETV6-ABL1 $(109,110)$. Notably, preclinical testing of small-molecule tyrosine kinase inhibitors (developed for the treatment of BCR-ABL1-positive leukemias) in NUP214-ABL1 tumors support the hypothesis that ABL1 inhibition may be used as a targeted therapy in these patients $(111,112)$.

Prototypical RAS-activating mutations that result in the accumulation of Ras in its active, GTP-bound conformation have been described in 5\%-10\% of T-ALLs, particularly in the early immature group $(32,33,113)$. In addition, cryptic deletions and/or mutations in the neurofibromatosis type 1 (NF1) gene, which encodes a negative regulator of the Ras pathway, occur in 3\% of T-ALL (114). Importantly, a conditional K-Ras ${ }^{\mathrm{G} 12 \mathrm{D}}$ murine knockin model, in which oncogenic K-Ras was expressed from its endogenous promoter, resulted in a highly penetrant, aggressive T cell leukemia/ lymphoma, further confirming an important role for RAS signaling in T cell transformation (115).
The IL-7 receptor signals through the JAK/STAT pathway and is strictly required to support the growth, proliferation, and survival of early $\mathrm{T}$ cell progenitor cells (116). Aberrant JAK signaling was first linked with T-ALL in the context of the $t(9 ; 12)(\mathrm{p} 24 ; \mathrm{p} 13)$ translocation, a rare rearrangement encoding the constitutively active ETV6-JAK2 kinase fusion oncoprotein (117). More recently, activating mutations in JAK1 and JAK3 have been reported in T-ALLs $(33,118,119)$. Moreover, somatic gain-of-function mutations in the $I L 7 R$ gene, encoding the IL-7 receptor and resulting in constitutive activation of JAK/STAT signaling, have recently been identified in approximately $10 \%$ of T-ALLs $(120,121)$.

\section{The challenge ahead: predicting prognosis and developing molecularly targeted drugs}

In conclusion, T-ALL is an aggressive hematologic cancer for which limited therapeutic options are available for patients with primary resistant or relapsed disease, underscoring the need for better treatment stratification protocols and for identifying more effective antileukemic drugs (2). This imperative is further supported by studies of the long-term effects of intensified chemotherapy in T-ALL survivors, which show that gains in leukemia-free survival have been achieved in parallel with significant increases in rates of acute and chronic life-threatening and debilitating toxicities (122).

The identification of activating NOTCH1 mutations that are present in over $60 \%$ of T-ALL patients (6) created enormous interest in developing molecularly tailored therapies for T-ALL and prompted the initiation of clinical trials to test the effectiveness of blocking NOTCH1 signaling with GSIs. The combination of GSIs and glucocorticoids may have increased efficacy and decreased toxicity in the treatment of T-ALL (123). In addition, the presence of activated kinase oncoproteins in a subset of T-ALLs may offer an additional opportunity for molecularly tailored therapies. Given the efficacy of ABL1 kinase inhibitors for the treatment of BCR-ABL1-positive leukemias and the sensitivity of NUP214-ABL1 to these inhibitors $(108,111$, 124), NUP214-ABL1-positive T-ALL patients may benefit from the inclusion of ABL1 inhibitors in their treatment schemes. Similarly, patients with activating JAK1 (119), JAK3 (33), or IL7R mutations $(120,121)$ might benefit from the JAK/STAT inhibitors currently under development for the treatment of myeloproliferative disorders.

Finally, understanding the pathogenesis of T-ALL is critical for the development of prognostic markers that may identify patients at increased risk of relapse. In light of this, recent studies have shown that early immature T-ALLs in children (31) and those with an absence of biallelic TCRG deletion (34) have a very poor prognosis. Intensive genetic characterization of these early immature leukemias has revealed great heterogeneity among these tumors (33). Nevertheless, these early immature leukemias share a gene expression signature closely related to hematopoietic stem cells and show overlapping myeloid and T-lymphoid immunophenotypic features and genetic alterations, suggesting that they may be more adequately treated with myeloid-based chemotherapy $(32,33)$.

Overall, the identification and molecular characterization of new oncogenes and tumor suppressors has uncovered much of the mechanisms involved in the pathogenesis of T-ALL. The development of representative and well-characterized xenograft and genetic animal models of T-ALL for preclinical testing, the identification of solid biomarkers of treatment response to stan- 
dard therapies, and the development of a dynamic framework of clinical trials that facilitates testing new and emerging drugs and drug combinations in the clinic are essential to ensure the effective translation of this information to the clinic in the form of molecularly tailored therapies for the treatment of T-ALL.
Address correspondence to: Adolfo Ferrando, Institute for Cancer Genetics, Columbia University, Irving Cancer Research Center 4-402A, 1130 St. Nicholas Ave., New York, New York 10032, USA. Phone: 212.851.4611; Fax: 212.851.5256; E-mail: af2196@ columbia.edu.
1. Ferrando AA, et al. Gene expression signatures define novel oncogenic pathways in T cell acute lymphoblastic leukemia. Cancer Cell. 2002;1(1):75-87.

2. Pui CH, Robison LL, Look AT. Acute lymphoblastic leukaemia. Lancet. 2008;371(9617):1030-1043.

3. Goldberg JM, et al. Childhood T-cell acute lymphoblastic leukemia: the Dana-Farber Cancer Institute acute lymphoblastic leukemia consortium experience. J Clin Oncol. 2003;21(19):3616-3622.

4. Oudot C, et al. Prognostic factors for leukemic induction failure in children with acute lymphoblastic leukemia and outcome after salvage therapy: the FRALLE 93 study. JClin Oncol. 2008;26(9):1496-1503.

5. Aifantis I, Raetz E, Buonamici S. Molecular pathogenesis of T-cell leukaemia and lymphoma. Nat Rev Immunol. 2008;8(5):380-390.

6. Weng AP, et al. Activating mutations of NOTCH1 in human $T$ cell acute lymphoblastic leukemia. Science. 2004;306(5694):269-271.

7. Hebert J, Cayuela JM, Berkeley J, Sigaux F. Candidate tumor-suppressor genes MTS1 (p16INK4A) and MTS2 (p15INK4B) display frequent homozygous deletions in primary cells from $\mathrm{T}$ - but not from B-cell lineage acute lymphoblastic leukemias. Blood. 1994;84(12):4038-4044.

8. Begley CG, et al. Chromosomal translocation in a human leukemic stem-cell line disrupts the T-cell antigen receptor delta-chain diversity region and results in a previously unreported fusion transcript. Proc Natl Acad Sci U S A. 1989;86(6):2031-2035.

9. Chen $\mathrm{Q}$, et al. The tal gene undergoes chromosome translocation in $\mathrm{T}$ cell leukemia and potentially encodes a helix-loop-helix protein. EMBO J. 1990; $9(2): 415-424$.

10. Bernard O, et al. Two distinct mechanisms for the SCL gene activation in the $\mathrm{t}(1 ; 14)$ translocation of T-cell leukemias. Genes Chromosomes Cancer. 1990; 1(3):194-208.

11. Xia Y, et al. TAL2, a helix-loop-helix gene activated by the $(7 ; 9)(\mathrm{q} 34 ; \mathrm{q} 32)$ translocation in human T-cell leukemia. Proc Natl Acad Sci U S A. 1991; 88(24):11416-11420.

12. Mellentin JD, Smith SD, Cleary ML. lyl-1, a novel gene altered by chromosomal translocation in $\mathrm{T}$ cell leukemia, codes for a protein with a helix-loophelix DNA binding motif. Cell. 1989;58(1):77-83.

13. Wang J, et al. The $\mathrm{t}(14 ; 21)$ (q11.2; 22$)$ chromosomal translocation associated with T-cell acute lymphoblastic leukemia activates the BHLHB1 gene. Proc Natl Acad Sci U S A. 2000;97(7):3497-3502.

14. Royer-Pokora B, Loos U, Ludwig WD. TTG-2, a new gene encoding a cysteine-rich protein with the LIM motif, is overexpressed in acute T-cell leukaemia with the $\mathrm{t}(11 ; 14)(\mathrm{p} 13 ; \mathrm{q} 11)$. Oncogene. 1991; 6(10):1887-1893.

15. McGuire EA, Hockett RD, Pollock KM, Bartholdi MF, O'Brien SJ, Korsmeyer SJ. The t $(11 ; 14)$ (p15;q11) in a T-cell acute lymphoblastic leukemia cell line activates multiple transcripts, including $\mathrm{Ttg}-1$, a gene encoding a potential zinc finger protein. Mol Cell Biol. 1989;9(5):2124-2132.

16. Boehm T, Foroni L, Kaneko Y, Perutz MF, Rabbitts $\mathrm{TH}$. The rhombotin family of cysteine-rich LIMdomain oncogenes: distinct members are involved in T-cell translocations to human chromosomes 11p15 and 11p13. Proc Natl Acad Sci U S A. 1991;88(10):4367-4371.

17. Dube ID, et al. A novel human homeobox gene lies at the chromosome 10 breakpoint in lymphoid neoplasias with chromosomal translocation t(10;14). Blood. 1991;78(11):2996-3003.
18. Hatano M, Roberts CW, Minden M, Crist WM, Korsmeyer SJ. Deregulation of a homeobox gene, HOX11, by the $\mathrm{t}(10 ; 14)$ in T cell leukemia. Science. 1991;253(5015):79-82.

19. Kennedy MA, et al. HOX11, a homeobox-containing T-cell oncogene on human chromosome 10q24. Proc Natl Acad Sci U S A. 1991;88(20):8900-8904.

20. Bernard OA, et al. A new recurrent and specific cryptic translocation, $\mathrm{t}(5 ; 14)(\mathrm{q} 35 ; \mathrm{q} 32)$, is associated with expression of the Hox11L2 gene in T acute lymphoblastic leukemia. Lenkemia. 2001; 15(10):1495-1504.

21. Homminga $\mathrm{I}$, et al. Integrated transcript and genome analyses reveal NKX2-1 and MEF2C as potential oncogenes in $\mathrm{T}$ cell acute lymphoblastic leukemia. Cancer Cell. 2011;19(4):484-497.

22. Nagel S, Kaufmann M, Drexler HG, MacLeod RA. The cardiac homeobox gene NKX2-5 is deregulated by juxtaposition with BCL11B in pediatric T-ALL cell lines via a novel $\mathrm{t}(5 ; 14)(\mathrm{q} 35.1 ; \mathrm{q} 32.2)$. Cancer Res. 2003;63(17):5329-5334.

23. Soulier J, et al. HOXA genes are included in genetic and biologic networks defining human acute T-cell leukemia (T-ALL). Blood. 2005;106(1):274-286.

24. Su X, et al. Transforming potential of the T-cell acute lymphoblastic leukemia-associated homeobox genes HOXA13, TLX1, and TLX3. Genes Chromosomes Cancer. 2006;45(9):846-855.

25. Erikson J, et al. Deregulation of c-myc by translocation of the alpha-locus of the T-cell receptor in T-cell leukemias. Science. 1986;232(4752):884-886.

26. Clappier E, et al. The C-MYB locus is involved in chromosomal translocation and genomic duplications in human T-cell acute leukemia (T-ALL), the translocation defining a new T-ALL subtype in very young children. Blood. 2007;110(4):1251-1261.

27. Ellisen LW, et al. TAN-1, the human homolog of the Drosophila notch gene, is broken by chromosomal translocations in $\mathrm{T}$ lymphoblastic neoplasms. Cell. 1991;66(4):649-661.

28. Aplan PD, Lombardi DP, Ginsberg AM, Cossman J, Bertness VL, Kirsch IR. Disruption of the human SCL locus by "illegitimate" V-(D)-J recombinase activity. Science. 1990;250(4986):1426-1429.

29 . Van Vlierberghe P, et al. The cryptic chromosomal deletion $\operatorname{del}(11)(\mathrm{p} 12 \mathrm{p} 13)$ as a new activation mechanism of LMO2 in pediatric T-cell acute lymphoblastic leukemia. Blood. 2006;108(10):3520-3529.

30. Van Vlierberghe P, et al. The recurrent SET-NUP214 fusion as a new HOXA activation mechanism in pediatric T-cell acute lymphoblastic leukemia. Blood. 2008;111(9):4668-4680.

31. Coustan-Smith E, et al. Early T-cell precursor leukaemia: a subtype of very high-risk acute lymphoblastic leukaemia. Lancet Oncol. 2009;10(2):147-156.

32. Van Vlierberghe P, et al. ETV6 mutations in early immature human T cell leukemias. J Exp Med. 2011; 208(13):2571-2579

33. Zhang J, et al. The genetic basis of early T-cell precursor acute lymphoblastic leukaemia. Nature. 2012;481(7380):157-163.

34. Gutierrez A, et al. Absence of biallelic TCRgamma deletion predicts early treatment failure in pediatric T-cell acute lymphoblastic leukemia. J Clin Oncol. 2010;28(24):3816-3823.

35. Greenwald I. LIN-12/Notch signaling: lessons from worms and flies. Genes Dev. 1998;12(12):1751-1762.

36. Sulis ML, et al. NOTCH1 extracellular juxtamembrane expansion mutations in T-ALL. Blood. 2008; 112(3):733-740.

37. O’Neil J, et al. FBW7 mutations in leukemic cells mediate NOTCH pathway activation and resistance to gamma-secretase inhibitors. J Exp Med. 2007;204(8):1813-1824.

38. Thompson BJ, et al. The SCFFBW7 ubiquitin ligase complex as a tumor suppressor in T cell leukemia. J Exp Med. 2007;204(8):1825-1835.

39. Lewis HD, et al. Apoptosis in T cell acute lymphoblastic leukemia cells after cell cycle arrest induced by pharmacological inhibition of notch signaling. Chem Biol. 2007;14(2):209-219.

40. Paganin M, Ferrando A. Molecular pathogenesis and targeted therapies for NOTCH1-induced Tcell acute lymphoblastic leukemia. Blood Rev. 2011; 25(2):83-90.

41. Milano J, et al. Modulation of notch processing by gamma-secretase inhibitors causes intestinal goblet cell metaplasia and induction of genes known to specify gut secretory lineage differentiation. Toxicol Sci. 2004;82(1):341-358.

42. van Es JH, et al. Notch/gamma-secretase inhibition turns proliferative cells in intestinal crypts and adenomas into goblet cells. Nature. 2005; 435(7044):959-963.

43. Moellering RE, et al. Direct inhibition of the NOTCH transcription factor complex. Nature. 2009; 462(7270):182-188.

44. Wu Y, et al. Therapeutic antibody targeting of individual Notch receptors. Nature. 2010; 464(7291):1052-1057.

45. Breit S, et al. Activating NOTCH1 mutations predict favorable early treatment response and longterm outcome in childhood precursor T-cell lymphoblastic leukemia. Blood. 2006;108(4):1151-1157.

46. van Grotel M, et al. The outcome of molecularcytogenetic subgroups in pediatric T-cell acute lymphoblastic leukemia: a retrospective study of patients treated according to DCOG or COALL protocols. Haematologica. 2006;91(9):1212-1221.

47. Asnafi $\mathrm{V}$, et al. JAK1 mutations are not frequent events in adult T-ALL: a GRAALL study. Br J Haematol. 2010;148(1):178-179.

48. Mansour MR, et al. Prognostic implications of NOTCH1 and FBXW7 mutations in adults with T-cell acute lymphoblastic leukemia treated on the MRC UKALLXII/ECOG E2993 protocol. J Clin Oncol. 2009;27(26):4352-4356.

49. Zuurbier L, et al. NOTCH1 and/or FBXW7 mutations predict for initial good prednisone response but not for improved outcome in pediatric T-cell acute lymphoblastic leukemia patients treated on DCOG or COALL protocols. Lenkemia. 2010;24(12):2014-2022.

50. Clappier E, et al. NOTCH1 and FBXW7 mutations have a favorable impact on early response to treatment, but not on outcome, in children with T-cell acute lymphoblastic leukemia (T-ALL) treated on EORTC trials 58881 and 58951. Lenkemia. 2010;24(12):2023-2031.

51. Kox C, et al. The favorable effect of activating NOTCH1 receptor mutations on long-term outcome in T-ALL patients treated on the ALL-BFM 2000 protocol can be separated from FBXW7 loss of function. Lenkemia. 2010;24(12):2005-2013.

52. Deftos ML, He YW, Ojala EW, Bevan MJ. Correlating notch signaling with thymocyte maturation. Immunity. 1998;9(6):777-786.

53. Real PJ, Ferrando AA. NOTCH inhibition and glucocorticoid therapy in T-cell acute lymphoblastic leukemia. Leukemia. 2009;23(8):1374-1377.

54. Samon JB, et al. Preclinical analysis of the gammasecretase inhibitor PF-03084014 in combination 
with glucocorticoids in T-cell acute lymphoblastic leukemia. Mol Cancer Ther. 2012;11(7):1565-1575.

55. Kamijo T, Weber JD, Zambetti G, Zindy F, Roussel MF, Sherr CJ. Functional and physical interactions of the ARF tumor suppressor with p53 and Mdm2. Proc Natl Acad Sci U S A. 1998;95(14):8292-8297.

56. Zhang Y, Xiong Y, Yarbrough WG. ARF promotes MDM2 degradation and stabilizes p53: ARF-INK4a locus deletion impairs both the $\mathrm{Rb}$ and $\mathrm{p} 53$ tumor suppression pathways. Cell. 1998;92(6):725-734.

57. Clappier E, et al. Cyclin D2 dysregulation by chromosomal translocations to TCR loci in T-cell acute lymphoblastic leukemias. Leukemia. 2006;20(1):82-86.

58. Remke $\mathrm{M}$, et al. High-resolution genomic profiling of childhood T-ALL reveals frequent copy-number alterations affecting the TGF-beta and PI3K-AKT pathways and deletions at $6 \mathrm{q} 15-16.1$ as a genomic marker for unfavorable early treatment response. Blood. 2009;114(5):1053-1062.

59. Mullighan CG, et al. Genome-wide analysis of genetic alterations in acute lymphoblastic leukaemia. Nature. 2007;446(7137):758-764.

60. Krieger D, et al. Frequency and clinical relevance of DNA microsatellite alterations of the CDKN2A/B, ATM and p53 gene loci: a comparison between pediatric precursor T-cell lymphoblastic lymphoma and T-cell lymphoblastic leukemia. Haematologica. 2010;95(1):158-162.

61. Bash RO, et al. Does activation of the TAL1 gene occur in a majority of patients with T-cell acute lymphoblastic leukemia? A pediatric oncology group study. Blood. 1995;86(2):666-676.

62. Kelliher MA, Seldin DC, Leder P. Tal-1 induces T cell acute lymphoblastic leukemia accelerated by casein kinase IIalpha. ЕMBOJ. 1996;15(19):5160-5166.

63. Condorelli GL, et al. T-cell-directed TAL-1 expression induces T-cell malignancies in transgenic mice. Cancer Res. 1996;56(22):5113-5119.

64. Ono Y, Fukuhara N, Yoshie O. TAL1 and LIM-only proteins synergistically induce retinaldehyde dehydrogenase 2 expression in T-cell acute lymphoblastic leukemia by acting as cofactors for GATA3. Mol Cell Biol. 1998;18(12):6939-6950.

65. O’Neil J, Shank J, Cusson N, Murre C, Kelliher M. TAL1/SCL induces leukemia by inhibiting the transcriptional activity of E47/HEB. Cancer Cell. 2004; 5(6):587-596.

66. Kikuchi A, et al. Clinical significance of TAL1 gene alteration in childhood T-cell acute lymphoblastic leukemia and lymphoma. Leukemia. 1993; 7(7):933-938.

67. Cave $\mathrm{H}$, et al. Clinical significance of HOX11L2 expression linked to $\mathrm{t}(5 ; 14)(\mathrm{q} 35 ; \mathrm{q} 32)$, of HOX11 expression, and of SIL-TAL fusion in childhood T-cell malignancies: results of EORTC studies 58881 and 58951. Blood. 2004;103(2):442-450

68. Bash RO, et al. Clinical features and outcome of T-cell acute lymphoblastic leukemia in childhood with respect to alterations at the TAL1 locus: a Pediatric Oncology Group study. Blood. 1993; 81(8):2110-2117.

69. Larson RC, et al. Protein dimerization between Lmo2 (Rbtn2) and Tal1 alters thymocyte development and potentiates $T$ cell tumorigenesis in transgenic mice. EMBO J. 1996;15(5):1021-1027.

70. Fisch P, et al. T-cell acute lymphoblastic lymphoma induced in transgenic mice by the RBTN1 and RBTN2 LIM-domain genes. Oncogene. 1992; 7(12):2389-2397.

71. McGuire EA, Rintoul CE, Sclar GM, Korsmeyer SJ. Thymic overexpression of Ttg- 1 in transgenic mice results in T-cell acute lymphoblastic leukemia/lymphoma. Mol Cell Biol. 1992;12(9):4186-4196.

72. Aplan PD, et al. An scl gene product lacking the transactivation domain induces bony abnormalities and cooperates with LMO1 to generate T-cell malignancies in transgenic mice. EMBO J. 1997; 16(9):2408-2419.
73. McCormack MP, et al. The Lmo2 oncogene initiates leukemia in mice by inducing thymocyte selfrenewal. Science. 2010;327(5967):879-883.

74. Dear TN, Sanchez-Garcia I, Rabbitts TH. The HOX11 gene encodes a DNA-binding nuclear transcription factor belonging to a distinct family of homeobox genes. Proc Natl Acad Sci U S A. 1993; 90(10):4431-4435.

75. Lu M, Gong ZY, Shen WF, Ho AD. The tcl-3 protooncogene altered by chromosomal translocation in T-cell leukemia codes for a homeobox protein. EMBO J. 1991;10(10):2905-2910.

76. Ferrando AA, et al. Prognostic importance of TLX1 (HOX11) oncogene expression in adults with T-cell acute lymphoblastic leukaemia. Lancet. 2004;363(9408):535-536.

77. De Keersmaecker K, et al. The TLX1 oncogene drives aneuploidy in $\mathrm{T}$ cell transformation. Nat Med. 2010;16(11):1321-1327.

78. Renneville A, et al. Wilms tumor 1 (WT1) gene mutations in pediatric T-cell malignancies. Leukemia. 2010;24(2):476-480.

79. Kleppe M, et al. Deletion of the protein tyrosine phosphatase gene PTPN2 in T-cell acute lymphoblastic leukemia. Nat Genet. 2010;42(6):530-535.

80. Tosello V, et al. WT1 mutations in T-ALL. Blood. 2009; 114(5):1038-1045.

81. Van Vlierberghe P, et al. PHF6 mutations in T-cell acute lymphoblastic leukemia. Nat Genet. 2010; 42(4):338-342.

82. Dadi S, et al. TLX homeodomain oncogenes mediate $\mathrm{T}$ cell maturation arrest in T-ALL via interaction with ETS1 and suppression of TCR $\alpha$ gene expression. Cancer Cell. 2012;21(4):563-576.

83. Ballerini $P$, et al. HOX11L2 expression defines a clinical subtype of pediatric T-ALL associated with poor prognosis. Blood. 2002;100(3):991-997.

84. Baak U, et al. Thymic adult T-cell acute lymphoblastic leukemia stratified in standard- and highrisk group by aberrant HOX11L2 expression: experience of the German multicenter ALL study group. Leukemia. 2008;22(6):1154-1160.

85. Ferrando AA, Look AT. Clinical implications of recurring chromosomal and associated molecular abnormalities in acute lymphoblastic leukemia. Semin Hematol. 2000;37(4):381-395.

86. Rubnitz JE, et al. Childhood acute lymphoblastic leukemia with the MLL-ENL fusion and $t(11 ; 19)$ (q23;p13.3) translocation. J Clin Oncol. 1999; 17(1):191-196

87. Asnafi V, et al. CALM-AF10 is a common fusion transcript in T-ALL and is specific to the TCRgammadelta lineage. Blood. 2003;102(3):1000-1006

88. Ferrando AA, et al. Gene expression signatures in MLL-rearranged T-lineage and B-precursor acute leukemias: dominance of HOX dysregulation. Blood. 2003;102(1):262-268.

89. Palomero T, et al. NOTCH1 directly regulates c-MYC and activates a feed-forward-loop transcriptional network promoting leukemic cell growth. Proc Natl Acad SciU S A. 2006;103(48):18261-18266.

90. Margolin AA, Palomero T, Sumazin P, Califano A, Ferrando AA, Stolovitzky G. ChIP-on-chip significance analysis reveals large-scale binding and regulation by human transcription factor oncogenes. Proc Natl Acad Sci U S A. 2009;106(1):244-249.

91. Lahortiga I, et al. Duplication of the MYB oncogene in T cell acute lymphoblastic leukemia. Nat Genet. 2007;39(5):593-595.

92. O'Neil J, et al. Alu elements mediate MYB gene tandem duplication in human T-ALL. J Exp Med. 2007; 204(13):3059-3066.

93. Gutierrez A, et al. Inactivation of LEF1 in T-cell acute lymphoblastic leukemia. Blood. 2010; 115(14):2845-2851.

94. Wang LC, et al. The TEL/ETV6 gene is required specifically for hematopoiesis in the bone marrow. Genes Dev. 1998;12(15):2392-2402.
95. Hock H, et al. Tel/Etv6 is an essential and selective regulator of adult hematopoietic stem cell survival. Genes Dev. 2004;18(19):2336-2341.

96. Bohlander SK. ETV6: a versatile player in leukemogenesis. Semin Cancer Biol. 2005;15(3):162-174.

97. Wakabayashi Y, et al. Bcl11b is required for differentiation and survival of alphabeta $T$ lymphocytes. Nat Immunol. 2003;4(6):533-539.

98. Gutierrez A, et al. The BCL11B tumor suppressor is mutated across the major molecular subtypes of T-cell acute lymphoblastic leukemia. Blood. 2011; 118(15):4169-4173.

99. Yamagata T, Maki K, Mitani K. Runx1/AML1 in normal and abnormal hematopoiesis. Int J Hematol. 2005;82(1):1-8.

100.Della Gatta G, et al. Reverse engineering of TLX oncogenic transcriptional networks identifies RUNX1 as tumor suppressor in T-ALL. Nat Med. 2012;18(3):436-440.

101.Grossmann V, et al. Prognostic relevance of RUNX1 mutations in T-cell acute lymphoblastic leukemia. Haematologica. 2011;96(12):1874-1877.

102.Ho IC, Tai TS, Pai SY. GATA3 and the T-cell lineage: essential functions before and after T-helper-2-cell differentiation. Nat Rev Immunol. 2009;9(2):125-135.

103. Ntziachristos P, et al. Genetic inactivation of the polycomb repressive complex 2 in T cell acute lymphoblastic leukemia. Nat Med. 2012;18(2):298-303.

104.Simon C, et al. A key role for EZH2 and associated genes in mouse and human adult T-cell acute leukemia. Genes Dev. 2012;26(7):651-656.

105. Cully M, You H, Levine AJ, Mak TW. Beyond PTEN mutations: the PI3K pathway as an integrator of multiple inputs during tumorigenesis. Nat Rev Cancer. 2006;6(3):184-192.

106. Palomero T, et al. Mutational loss of PTEN induces resistance to NOTCH1 inhibition in T-cell leukemia. Nat Med. 2007;13(10):1203-1210.

107. Hagemeijer A, Graux C. ABL1 rearrangements in T-cell acute lymphoblastic leukemia. Genes Chromosomes Cancer. 2010;49(4):299-308.

108. Graux C, et al. Fusion of NUP214 to ABL1 on amplified episomes in T-cell acute lymphoblastic leukemia. Nat Genet. 2004;36(10):1084-1089.

109. De Keersmaecker K, et al. Fusion of EML1 to ABL1 in T-cell acute lymphoblastic leukemia with cryptic t(9;14)(q34;q32). Blood. 2005;105(12):4849-4852.

110.Van Limbergen $\mathrm{H}$, et al. Molecular cytogenetic and clinical findings in ETV6/ABL1-positive leukemia. Genes Chromosomes Cancer. 2001;30(3):274-282.

111. Quintas-Cardama A, et al. Activity of tyrosine kinase inhibitors against human NUP214-ABL1-positive T cell malignancies. Leukemia. 2008;22(6):1117-1124.

112.De Keersmaecker K, et al. Kinase activation and transformation by NUP214-ABL1 is dependent on the context of the nuclear pore. Mol Cell. 2008; 31(1):134-142.

113. Bar-Eli M, Ahuja H, Foti A, Cline MJ. N-RAS mutations in T-cell acute lymphocytic leukaemia: analysis by direct sequencing detects a novel mutation. Br J Haematol. 1989;72(1):36-39.

114. Balgobind BV, et al. Leukemia-associated NF1 inactivation in patients with pediatric T-ALL and AML lacking evidence for neurofibromatosis. Blood. 2008; 111(8):4322-4328.

115.Kindler T, et al. K-RasG12D-induced T-cell lymphoblastic lymphoma/leukemias harbor Notch1 mutations and are sensitive to gamma-secretase inhibitors. Blood. 2008;112(8):3373-3382.

116. Mazzucchelli R, Durum SK. Interleukin-7 receptor expression: intelligent design. Nat Rev Immunol. 2007;7(2):144-154

117. Lacronique $\mathrm{V}$, et al. A TEL-JAK2 fusion protein with constitutive kinase activity in human leukemia. Science. 1997;278(5341):1309-1312.

118. Asnafi $\mathrm{V}$, et al. JAK1 mutations are not frequent events in adult T-ALL: a GRAALL study. $\mathrm{Br} \mathrm{J} \mathrm{Hae-}$ matol. 2010;148(1):178-179. 
119. Flex E, et al. Somatically acquired JAK1 mutations in adult acute lymphoblastic leukemia. J Exp Med. 2008;205(4):751-758.

120. Shochat C, et al. Gain-of-function mutations in interleukin-7 receptor-alpha (IL7R) in childhood acute lymphoblastic leukemias. J Exp Med. 2011; 208(5):901-908.

121.Zenatti PP, et al. Oncogenic IL7R gain-of-function mutations in childhood T-cell acute lymphoblastic leukemia. Nat Genet. 2011;43(10):932-939.

122. Barrett AJ, et al. Bone marrow transplants from HLA-identical siblings as compared with chemotherapy for children with acute lymphoblastic leukemia in a second remission. N Engl J Med. 1994; 331(19):1253-1258.

123. Real PJ, et al. Gamma-secretase inhibitors reverse glucocorticoid resistance in $\mathrm{T}$ cell acute lymphoblastic leukemia. Nat Med. 2009;15(1):50-58.

124.De Keersmaecker K, Versele M, Cools J, Superti-Furga G, Hantschel O. Intrinsic differences between the catalytic properties of the oncogenic NUP214-ABL1 and BCR-ABL1 fusion protein kinases. Leukemia. 2008;22(12):2208-2216.

125. Ferrando AA. The role of NOTCH1 signaling in T-ALL. Hematology Am Soc Hematol Educ Program. 2009;2009:353-361.

126.van Grotel M, et al. Prognostic significance of molecular-cytogenetic abnormalities in pediatric T-ALL is not explained by immunophenotypic differences. Leukemia. 2008;22(1):124-131.

127. Gottardo NG, Jacoby PA, Sather HN, Reaman GH, Baker DL, Kees UR. Significance of HOX11L2/ TLX3 expression in children with T-cell acute lymphoblastic leukemia treated on Children's Cancer Group protocols. Leukemia. 2005;19(9):1705-1708.

128. Speleman $\mathrm{F}$, et al. A new recurrent inversion, $\operatorname{inv}(7)(\mathrm{p} 15 \mathrm{q} 34)$, leads to transcriptional activation of HOXA10 and HOXA11 in a subset of T-cell acute lymphoblastic leukemias. Lenkemia. 2005; 19(3):358-366

129.Asnafi V, et al. NOTCH1/FBXW7 mutation identifies a large subgroup with favorable outcome in adult T-cell acute lymphoblastic leukemia (T-ALL): a Group for Research on Adult Acute
Lymphoblastic Leukemia (GRAALL) study. Blood. 2009;113(17):3918-3924.

130.Gutierrez A, et al. High frequency of PTEN, PI3K, and AKT abnormalities in T-cell acute lymphoblastic leukemia. Blood. 2009;114(3):647-650.

131. Burmeister T, Gokbuget N, Reinhardt R, Rieder $\mathrm{H}$, Hoelzer D, Schwartz S. NUP214-ABL1 in adult T-ALL: the GMALL study group experience. Blood. 2006;108(10):3556-3559.

132. Raanani $\mathrm{P}$, et al. Philadelphia-chromosome-positive T-lymphoblastic leukemia: acute leukemia or chronic myelogenous leukemia blastic crisis. Acta Haematol. 2005;113(3):181-189.

133.Paietta E, et al. Activating FLT3 mutations in CD117/KIT(+) T-cell acute lymphoblastic leukemias. Blood. 2004;104(2):558-560.

134.Van Vlierberghe P, et al. Activating FLT3 mutations in CD4+/CD8- pediatric T-cell acute lymphoblastic leukemias. Blood. 2005;106(13):4414-4415.

135. Ntziachristos $\mathrm{P}$, et al. Genetic inactivation of the polycomb repressive complex 2 in T cell acute lymphoblastic leukemia. Nat Med. 2012;18(2):298-301. 